\title{
Editorial
}

\section{Application of Discrete Mathematics in Urban Transportation System Analysis}

\author{
Baozhen Yao, ${ }^{1}$ Bin Yu, ${ }^{2}$ Gang Chen, ${ }^{3}$ Rui Mu, ${ }^{4}$ and Fang Zong ${ }^{5}$ \\ ${ }^{1}$ School of Automotive Engineering, Dalian University of Technology, Dalian 116024, China \\ ${ }^{2}$ Transportation Management College, Dalian Maritime University, Dalian 116024, China \\ ${ }^{3}$ Department of Mechanical and Manufacturing Engineering, Aalborg University, Denmark \\ ${ }^{4}$ Faculty of Technology, Policy and Management, Delft University of Technology, Delft, The Netherlands \\ ${ }^{5}$ College of Transportation, Jilin University, Changchun 30022, China
}

Correspondence should be addressed to Bin Yu; ybzhyb@163.com

Received 5 May 2014; Accepted 5 May 2014; Published 15 June 2014

Copyright (C) 2014 Baozhen Yao et al. This is an open access article distributed under the Creative Commons Attribution License, which permits unrestricted use, distribution, and reproduction in any medium, provided the original work is properly cited.

Urban transportation system is of foremost importance to support the passengers and freight mobility requirements of urban agglomerations. Transportation in urban areas is highly complex because of the modes involved, the multitude of origins and destinations, and the amount and variety of traffic. Analysing the urban transportation system is the process of attempting to examine the behaviour patterns of the elements of urban transportation system and the interactions among them, which is very critical for urban traffic planning and management. Discrete mathematics is the branch of mathematics dealing with objects that can be assumed by only distinct, separated values. Since the behaviour of travellers and vehicles, which are the main elements in urban transportation system, is in an individual level, compared with traditional aggregated methods, the techniques of discrete mathematics are more suitable to be adopted in urban transportation system analysis. Therefore, discrete mathematics is gradually becoming the popular method to analyse transportation system and has attracted much attention of the researchers.

Within this context, this special issue serves as a forum to highlight the most significant recent developments in the techniques of discrete mathematics, especially discrete choice models, Bayesian network, and so on, and to apply these techniques on urban transportation system analysis, such as travel behaviour, taxi's driving pattern, and delivery route selection.

Some works focus on the application of prediction in urban transportation system; for example, Z. Mingheng et al's work "Accurate multisteps traffic flow prediction based on SVM" attempted to use support vector machine to predict traffic flow for intelligent traffic management. In the prediction method, a multisteps prediction was adopted to improve the performance of prediction. The results indicated that the proposed support vector machine had a good ability for traffic flow prediction. F. Zong et al.s paper "Prediction for traffic accident severity: comparing the Bayesian network and regression models" tried to predict traffic accident severity by comparing the Bayesian network and regression models. In their paper, there were three severity indicators, that is, number of fatalities, number of injuries, and property damage, which were used. L. Li et al. "Deterioration prediction of urban bridges on network levelusing Markov-chain model" aimed to predict urban bridge deterioration with Markovchain model. In the model, three deterioration circumstances were considered, and the results showed that recoverable repair treatments were important for bridge condition and the prediction method was effective for predicting bridge deterioration. F. Zong et al.s work "Predicting severity and duration of road traffic accident" presented a model system to predict the severity and duration of road traffic accidents. In the model, ordered probit model and hazard model were used, respectively. The results suggested that the ordered probit model had a higher prediction performance than the SVM model.

Some researchers focused on the detection or assessment problem in transportation system. Y. Bao et al's paper "Structural damage identification of pipe based on GA and 
SCE-UA algorithm" wanted to identify structural damage effectively. They proposed genetic algorithm and SCE-UA algorithm to detect platform structure damage in their paper. The results showed that the two algorithms have high identification accuracy and good adaptability. B. Peng et al.s paper "Static and dynamic analysis of railway reinforced system with cross-beams" proposed a static and dynamic analysis of railway reinforced system with cross-beams. Y. Bao et al's work "Damage detection of bridge structure based on SVM" attempted to detect the damage of bridge pier for bridge management and maintenance. In their paper, support vector machine was used to detect the damage of bridge pier. The results suggested that the support vector machine was an effective method for detecting damage of bridge pier. $\mathrm{H}$. Wang and J. Gao's work "Bayesian network assessment method for civil aviation safety based on flight delays" proposed a Bayesian network assessment method for civil aviation safety. In the model, the Bayesian network was used to build the aviation operation safety assessment based on flight delay. B. Yan et al's work "Beam structure damage identification based on BP neural network and support vector machine" attempted to use BP neural network and support vector machine to identify beam structure damage. The results showed that the two methods had a preferable identification precision.

Also assignment methods in urban transportation system are discussed in some works. Y. Jiang et al.s work "Multiobjective gate assignment based on passenger walking distance and fairness" attempted to shorten the walking distance and balance the airlines' service quality by using a multiobjective gate assignment model. Test results indicated that the optimization model could reduce effectively the walking distance of passenger and improve the number of flights. H. Wang et al's work "Real-time gate reassignment based on flight delay featurein hub airport" tried to achieve real-time gate reassignment in hub airport. The objective was to minimize the disturbance led by gate reassignment based flight delay feature and ant colony algorithm was used to solve the problem.

There are also some works on the planning problem in this field. W. Juan et al.s work "The best path analysis in military highway transport based on DEA and multiobjective fuzzy decision-making" presented data envelopment analysis and multiobjective fuzzy decision-making to select the best path for military highway transport. L. Guo et al's work "Lane changing trajectory planning and tracking controller design for intelligent vehicle running on curved road" tried to enhance the active safety and presented lane changing trajectory planning and tracking controller design for intelligent vehicle running on curved road. Z. Yaobao et al's work "An improved particle swarm optimization for the automobile spare part warehouse location problem" used a particle swarm optimization to solve the automobile spare part warehouse location problem. In the algorithm, acceleration coefficients and crossover operation were used to improve the performance of the algorithm.

Travel behaviour and weaving behaviour are also considered in some papers. L. Sun et al.'s work "Study on the weaving behavior of high density bidirectional pedestrian flow" discussed the characteristic of pedestrian weaving behaviour. In the study, video analysis was selected to extract pedestrian moving behaviour. L. Gao et al's work "The effect of travel information on travelers' choice of travel modes and routes: a case study of the travel between the campuses" attempted to study the travelers' choices of travel modes and routes. In their paper, a case study of the travel between Minhang campus and Xuhui campus of Shanghai Jiao Tong University was conducted. C. Zhuge et al's work "An initial implementation of multiagent simulation of travelbehavior for a mediumsized city in China" proposed an agent-based simulation of travel behaviour to acquire the travel behaviour features in a medium-sized city in China.

Other methods like transport turnover and vessel collision avoidance strategy are also studied in this special issue. Z. Yang et al's paper "Transport turnover with spatial econometric perspective under the energy conservation and emissions reduction in China" proposed a spatial econometric model to solve the space correlation of road turnover among 31 provinces in China. Q. Xu et al.'s work "Multiobjective optimization based vessel collision avoidance strategy optimization" presented a multiobjective optimization to reduce the human fault and improve the safety of marine traffic. And a multiobjective optimization algorithm NSGA-II was used to solve the problem.

These articles demonstrate the advancement of the recent development in techniques like discrete choice models, Bayesian network, and support vector machine in urban transportation system.

\section{Acknowledgments}

We would like to express our gratitude to the many reviewers for their hard work. We would also like to thank the authors for their contributions to the special issue. This special issue could not have been completed without their dedication and support.

Baozhen Yao Bin $Y u$

Gang Chen Rui $M u$

Fang Zong 


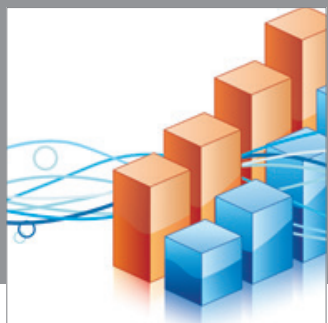

Advances in

Operations Research

mansans

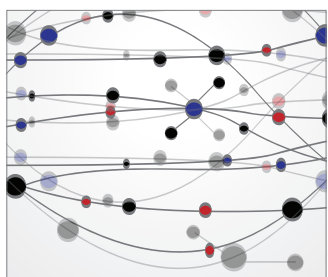

The Scientific World Journal
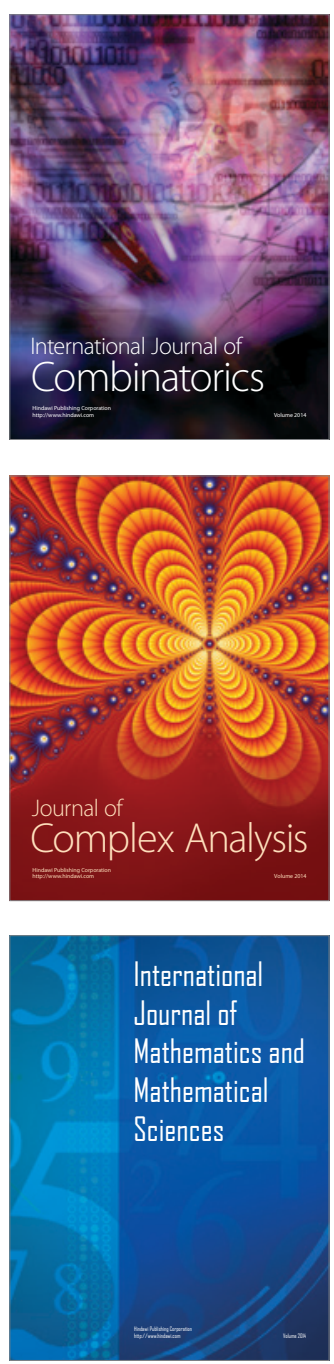
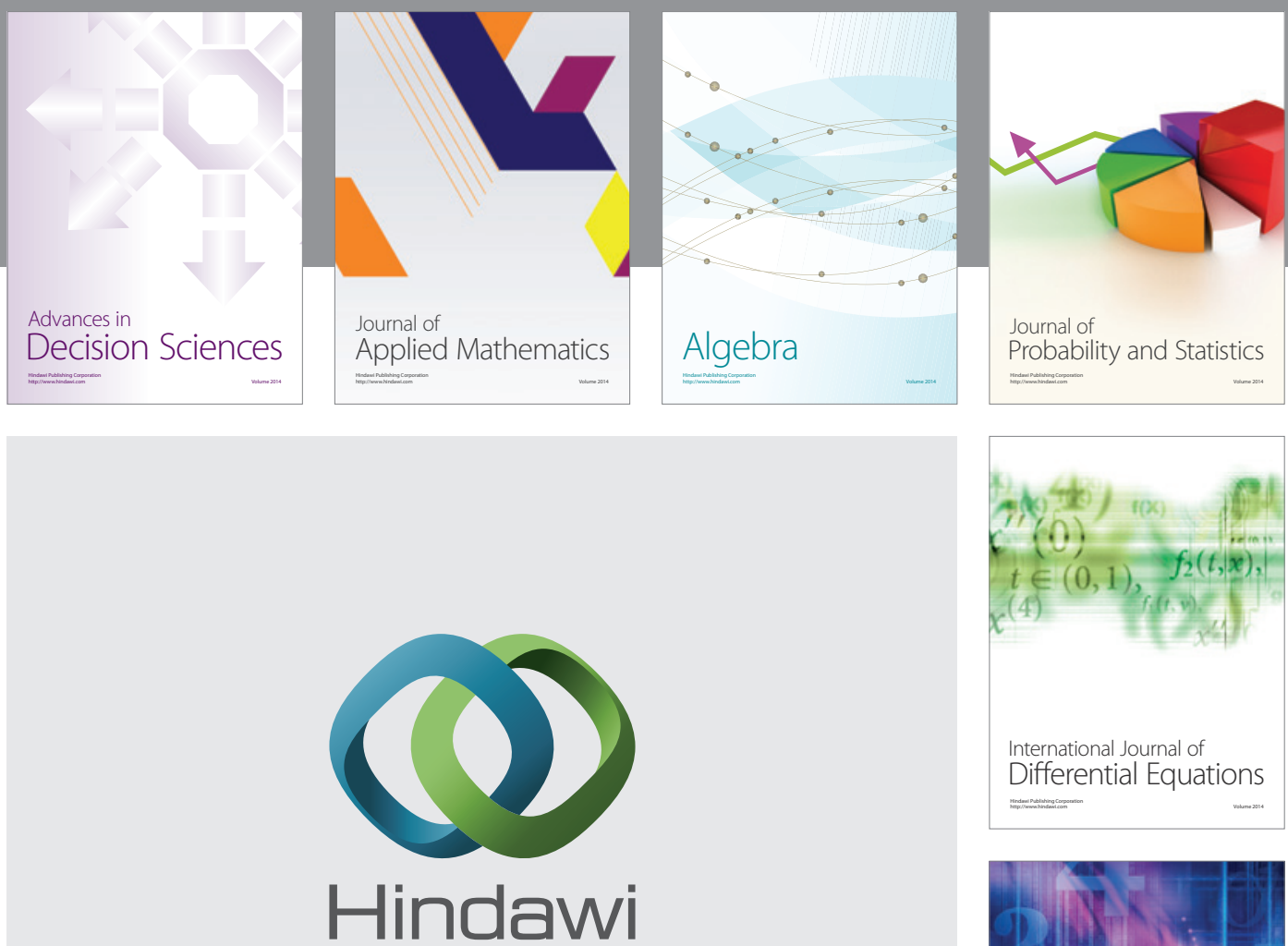

Submit your manuscripts at http://www.hindawi.com
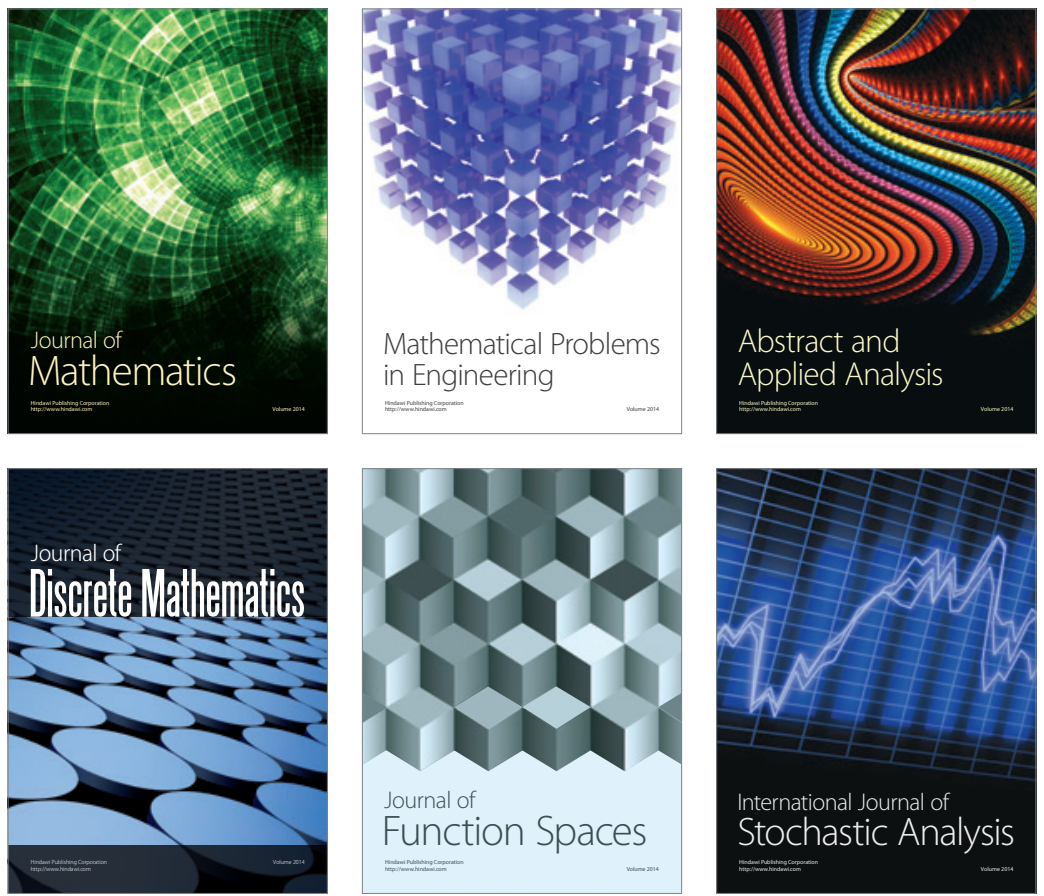

Journal of

Function Spaces

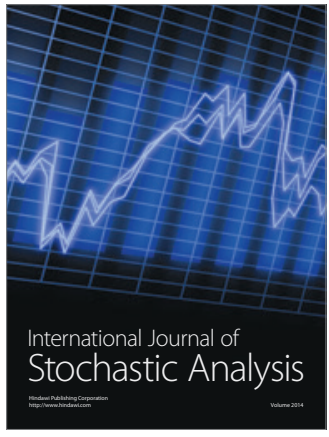

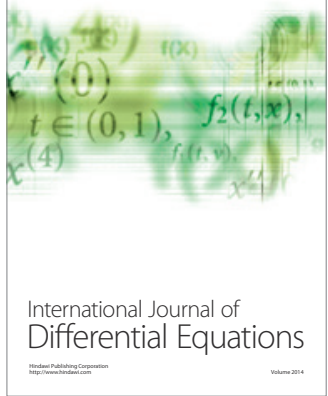
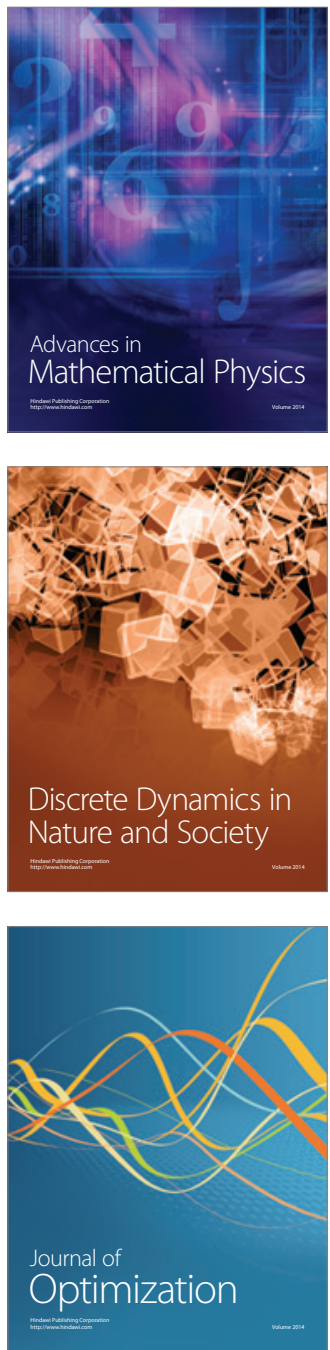